\title{
In-situ Experiments to Capture the Evolution of Microstructure During Phase Transformation of Titanium Under Dynamic Loading
}

\author{
Benjamin M. Morrow ${ }^{1, *}$, David R. Jones ${ }^{1}$, Paulo A. Rigg ${ }^{2}$, George T. Gray, III ${ }^{1}$, and Ellen K. Cerreta ${ }^{1}$ \\ ${ }^{1}$ Los Alamos National Laboratory \\ ${ }^{2}$ Dynamic Compression Sector (DCS), Institute for Shock Physics, Washington State University, Argonne National Laboratory
}

\begin{abstract}
Under sufficient stresses, such as during dynamic loading, titanium experiences a phase transformation from hcp alpha phase to hexagonal omega phase. Omega phase is often retained in the microstructure after unloading, and has a strong influence on subsequent mechanical properties. Simulations suggest there are multiple pathways and underlying mechanisms for this transformation. Due to the incredibly short timescales involved, experimental measurements for model validation have been difficult. However, new capabilities at the Advanced Photon Source have enabled diffraction measurements during plate impact experiments to study the evolution of titanium during transformation. These high-rate data allow us to probe the mechanism and kinetics of phase transformations in new ways. Recent results will be presented and compared to post-mortem characterization of soft-recovered shocked specimens. Comparisons are made with previous tests where material was shock-loaded and soft recovered for microstructural analysis. Together these techniques create a consistent picture of material behavior during the shock-induced $\alpha-\omega$ phase transformation in titanium.
\end{abstract}

\section{Introduction}

The solid-solid phase transition between hexagonal closepacked (hcp) alpha and hexagonal omega phases in titanium has important implications on mechanical properties. Omega phase can form in hcp metals under shock loading conditions, and, as a metastable phase at ambient pressure, can be retained in the microstructure after unloading, where it can contribute to subsequent deformation behavior [1-5]. Previous studies have examined the shock response of Ti single and multicrystals [6], and polycrystals [7-9]. Post-mortem microstructural investigations are an essential piece to determination of phase transformation pathways, but can only infer likely operations based on beginning and end states. In previous work, it was observed that when shock-loaded to $\sim 15 \mathrm{GPa}$, titanium forms a twophase microstructure, with characteristic omega lathes inside alpha grains (suggesting a predominately forward, $\alpha \rightarrow \omega$ pathway where the grains never reach $100 \% \omega-$ phase). It also suggested that the observed material behavior during shock can be described by the complex interplay between traditional plasticity and phase transformation mechanisms. Like twinning, it is expected that the $\alpha-$ $\omega$ phase transformation is a process characterized by both nucleation and growth. However, such post-mortem analyses are not well-suited to measure order of operations, relative activity of mechanisms during testing, or kinetics. Hence, in-situ X-ray diffraction (XRD) is required to obtain a complete picture of deformation behavior and phase transformations during shock loading. Recent advances in in-situ characterization techniques, including shock test-

\footnotetext{
*e-mail: morrow@lanl.gov
}

ing at advanced light sources, have unlocked the ability to directly observe microstructural processes and resulting material states during loading [10]. The current work examines the $\alpha-\omega$ phase transition in titanium using XRD during plate impact shock loading.

\section{Experimental Procedures}

\subsection{Testing}

High-purity $(99.995 \%)$ polycrystalline titanium was used for this study. The material chemistry and processing was the same as a previous study [7]. Briefly, the material was supplied by American Elements (USA) as $20 \mathrm{~mm}$ thick plate. The plate was rolled and annealed in steps to reduce initial grain size. Samples were machined from the plate using electrical discharge machining and annealed under vacuum at $873 \mathrm{~K}$ for 4 hours. The oxygen impurity content is less than $80 \mathrm{ppm}$. Full chemical analysis is shown in Table 1.

A strong $c$-axis fiber texture aligned with the plate normal (through-thickness (TT) direction) was present in the specimens, and the microstructure consists of twinfree equiaxed grains with a $21-27 \mu \mathrm{m}$ average grain size. Cylindrical specimens in form of $150 \mu \mathrm{m}$ thick foils were prepared with faces parallel to the TT direction. Each thinned specimen was backed with a LiF window to hold the material at pressure for an extended period of time before release.

The specimen was fixed over a hole on a brass holder and bolted to a larger target assembly to interface with the 
Table 1. Chemical composition of as-received titanium plate, in ppm [7].

\begin{tabular}{lccccccccccc}
\hline Element & $\mathrm{Fe}$ & $\mathrm{V}$ & $\mathrm{Al}$ & $\mathrm{Si}$ & $\mathrm{Ni}$ & $\mathrm{Mn}$ & $\mathrm{Cr}$ & $\mathrm{Cu}$ & $\mathrm{Mg}$ & $\mathrm{O}$ & $\mathrm{Ti}$ \\
\hline Amount $(\mathrm{ppm})$ & 10.7 & 1.1 & 3.6 & 11.6 & 3.2 & 1.2 & 2.6 & 1.5 & 2.5 & $<80$ & Balance \\
\hline
\end{tabular}

gun barrel inside a vacuum catch tank. Diagnostics behind the target looking through the backing window, notably photon Doppler velocimetry (PDV) and Velocimetry Interferometer System for Any Reflector (VISAR) probes, enabled velocity measurements of the interface between $\mathrm{Ti}$ and the LiF window. Shock loading was performed using the Impact Facilities at the Dynamic Compression Sector (DCS) at the Advanced Photon Source (APS) at Argonne National Laboratory. A catch tank downrange of the original sample location was fitted with steel plates to arrest debris after impact, and no recovery of samples for postmortem examination was possible. Figure 1 shows the experimental setup (with the vacuum tank open).

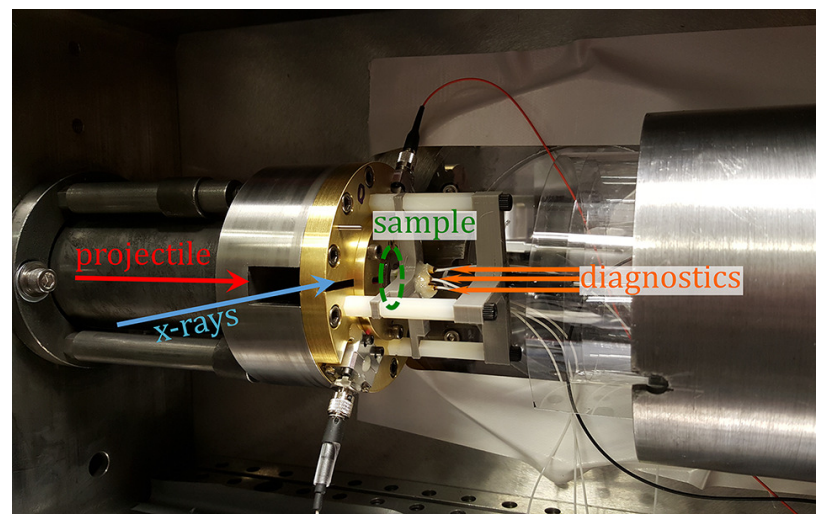

Fig. 1. Experimental setup of single-stage powder gun at DCS. The brass target plate containing the sample is fixed to the end of the powder gun (left). Diagnostics are attached to the back side of the sample. During testing, the projectile moves down the gun barrel (left), strikes the sample while x-rays are transmitted (center), and debris is collected in the back of the tank (right).

A LiF impactor was propelled down the barrel of a single stage powder gun towards each sample. A laser near the sample was used as a light gate to detect the incoming projectile and trigger the $\mathrm{x}$-rays and corresponding CCD image frames. X-rays were transmitted at a $25^{\circ}$ angle through the front (impact) surface of the sample. Transmission Laue diffraction was used to create diffraction patterns, which were collected on a detector system comprised of a $\sim 75 \mathrm{~mm}$ scintillator, intensifier, splitter, and four PiMax cameras triggered to coincide with incoming X-rays [11]. The synchrotron X-ray beam was supplied in bunches spaced 153 ns apart. A U27 electromagnetic undulator was used to supply pink beam radiation with the third harmonic tuned to $23 \mathrm{keV}$. Four $\mathrm{Rh}$ mirrors and two filters (Al and Ag) were used to isolate the third harmonic. Distance between the sample and detector was set to the minimum possible length allowed by the experimental setup $(\sim 160 \mathrm{~mm})$, and the $\mathrm{x}$-ray beam center was offset to one edge of the detector, to capture the largest amount of diffraction space possible.

\subsection{Data Analysis Methods}

X-ray diffraction patterns were collected during shock loading as described above. Images were first corrected to account for distortions in the imaging system and to align corresponding pixels between frames (which depict the same scintillator viewed by separate cameras). The Dioptas software package [12] was used to analyze XRD images. Polycrystalline silicon was used as a standard material to calibrate images to allow quantification of the d-spacings of visible planes in diffraction patterns. Initial sample-to-detector distance for the static (pre-shock) samples was calculated from the standard, also. Because the samples move downrange after impact, true sampleto-detector distance for each independent frame was determined by imposing a geometric correction that accounted for the time relative to impact and the sample velocity at that time. Bright $\mathrm{LiF}$ peaks originating from the impactor and backing window were masked and omitted. Intensity was integrated radially about the transmitted spot to convert XRD rings into peaks for line profile analysis and easier comparison to tabulated d-spacings.

\section{Results and Discussion}

\subsection{Shock Conditions \& Velocimetry}

Table 2 shows the shot matrix used in this study. Both tests were carried out using the same shock conditions with different timing to capture early (16-1-003) and late-stage (16-1-002) material behavior. Velocity at the Ti-LiF inter-

Table 2. Matrix of testing performed at DCS, including projectile velocity and resulting stresses in peak and steady-state shock states (Shock State 1 and 2, respectively).

\begin{tabular}{cccc}
\hline Test ID & $\begin{array}{c}\text { Projectile } \\
\text { Velocity } \\
(\mathrm{km} / \mathrm{s})\end{array}$ & $\begin{array}{c}\text { Shock } \\
\text { State 1 } \\
(\mathrm{GPa})\end{array}$ & $\begin{array}{c}\text { Shock } \\
\text { State 2 } \\
(\mathrm{GPa})\end{array}$ \\
\hline $16-1-002$ & 1.647 & 16.6 & 12.5 \\
$16-1-003$ & 1.625 & 16.6 & 12.5 \\
\hline
\end{tabular}

face was measured using VISAR. The Rankine-Hugoniot equations were used to determine stress from VISAR as outlined elsewhere [7], and is shown in Figure 2. After impact, the titanium loaded to shock state 1 (16.6 GPa), where it remained for approximately $50 \mathrm{~ns}$, until partial release from the rear $\mathrm{LiF}$ window dropped the stress to shock state $2(12.5 \mathrm{GPa})$. The sample remained at stress state 2 until unloading, about 700 ns after impact. X-ray bunches were spaced $153 \mathrm{~ns}$ apart in time at fixed intervals. To account for the effect of system jitter, the XRD frames triggered coincident with the X-ray bunch nearest to impact time. Because of this, it is impossible to prespecify the image timings relative to impact with greater 


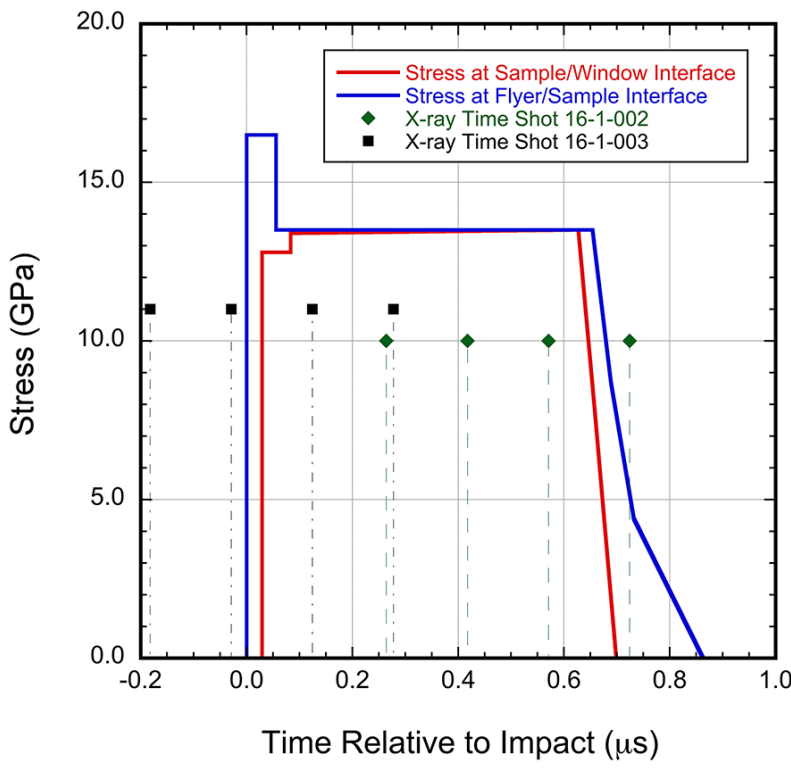

Fig. 2. Stress at the flyer-Ti and Ti-LiF surfaces as a function of time after impact, calculated using a 1-D shock-wave code [7]. Timings for the XRD camera frames are overlaid.

accuracy than $\sim 76 \mathrm{~ns}$, making it unlikely that shock state 1 will be sampled. Even so, timing relative to impact can be exactly calculated after the tests to determine the state of stress in the sample during each frame. Projectile velocity was chosen to ensure that the stress during shock state 2 would be greater than $10.1 \mathrm{GPa}$, the $\alpha-\omega$ transition stress for high-purity Ti [7]. This stress is also similar to previous tests where material was shocked and soft recovered for microstructural analysis so that the present diffraction technique would be directly comparable to previous work [6-8].

\subsection{Diffraction Analysis}

Figure 3 shows the XRD patterns obtained before and during shock-loading. Both tests displayed similar behavior, so for simplicity, the analysis is shown for test 16-1-002 only. As shown in Figure 2, with the addition on the ambient frame, this single test shows the entire lifecycle of titanium including unloaded, shocked, and partially unloaded states.

The ambient frame exhibits many distinct spots, each corresponding to a strongly diffracting plane in a single grain. Spots from many grains form characteristic rings with their centers on the beam center (at the left of the pattern), with each ring corresponding to a major plane. As each spot corresponds to a different grain orientation, this indicates that there are on the order of tens or hundreds of grain orientations being sampled initially (before shock).

Line profiles showing integrated intensities of select frames are shown in Figure 4. For simplicity, each subfigure represents a comparison between two material states. The first image (Figure 4a) compares ambient and shocked states. The center image (Figure 4b) compares shocked and partially released states. The third image (Figure 4c) compares the ambient and partially released states.

Several peaks are evident in the ambient curve, indexing to $\alpha$-Ti. Because the $\mathrm{x}$-ray beam is not monochromatic, peaks appear with some thickness, and have a larger tail to one side. Pink beam synchrotron radiation has the benefit of offering a slightly wider range of conditions to allow grains to diffract strongly and hence be captured by the detector. With knowledge of the x-ray beam character, the shape and location of diffraction peaks can be simulated for comparison with experimentally measured peaks. For the present work, analysis was performed such that the peak maxima were coincident with expectations for the predominant beam energy $(23 \mathrm{keV})$. The shape of the peaks was not used for further analysis. The observed d-spacings for the peak maxima are slightly shifted, indicating that some heating due to interaction with the x-ray beam has occurred. Rough estimates based on both in-situ annealing experiments and geometric attenuation of $\mathrm{Ti}$ in a similar setup as used here indicate a temperature rise on the order of $170{ }^{\circ} \mathrm{C}$. This temperature is fairly low relative to the melting temperature of $\mathrm{Ti}$, so it is expected to have only a small impact on the mechanical behavior. A new heat load chopper was added to subsequent tests at the beamline to reduce $\mathrm{x}$-ray time-on-sample to eliminate beam heating in the future. For the present work, the effect of temperature on d-spacing, assumed as a small, systematic offset, was taken into account when identifying peaks.

During shock loading, an immediate refinement of the microstructure is seen. This manifests in Figure 3 as a change from distinct spots to smooth rings (denoted by arrows in Frame 1). Previous post-mortem analysis of shocked and recovered material confirms that each alpha grain becomes fragmented by the nucleation and growth of several variants of omega [7,8]. As a result of this fragmentation, effective grain size is greatly reduced, increasing the number of orientations being sampled into the thousands, leading to smoother diffraction rings. One ring, in particular, is especially prominent in both the shocked XRD patterns and the line profile for $\mathrm{t}=264 \mathrm{~ns}$. Upon close inspection, this peak contains features beyond what is expected from diffraction from a single plane, indicating that multiple closely spaced planes, possibly from different phases, are present in a single peak. Indexing of this ring indicates that this is consistent with both compressed $\alpha$-Ti and newly formed $\omega$-Ti. The microstructure required to generate this signal is also consistent with microstructures from previously recovered samples. Due to the complexity of the experiments, the XRD patterns and resulting line profiles are not currently suitable for more detailed analysis, such as Rietveld refinement techniques used commonly in static diffraction studies [5]. Overall, pattern intensity tends to decrease after impact as the beam is partially attenuated by the LiF impactor. This increases noise and suppresses weaker peaks, making it infeasible to determine phase fraction using standard line profile analysis. Additionally, several large, bright peaks are evident in Figure 3 during shock. These are diffraction peaks for the LiF impactor. These large peaks were masked and omit- 


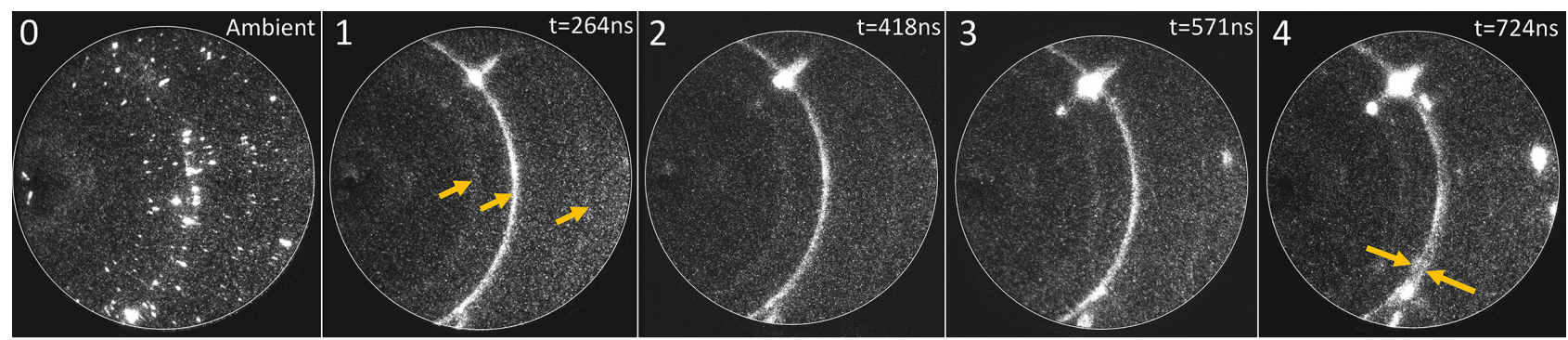

Fig. 3. X-ray diffraction patterns for high-purity titanium before and during shock loading. X-ray data was collected at the times indicated in Figure 2.
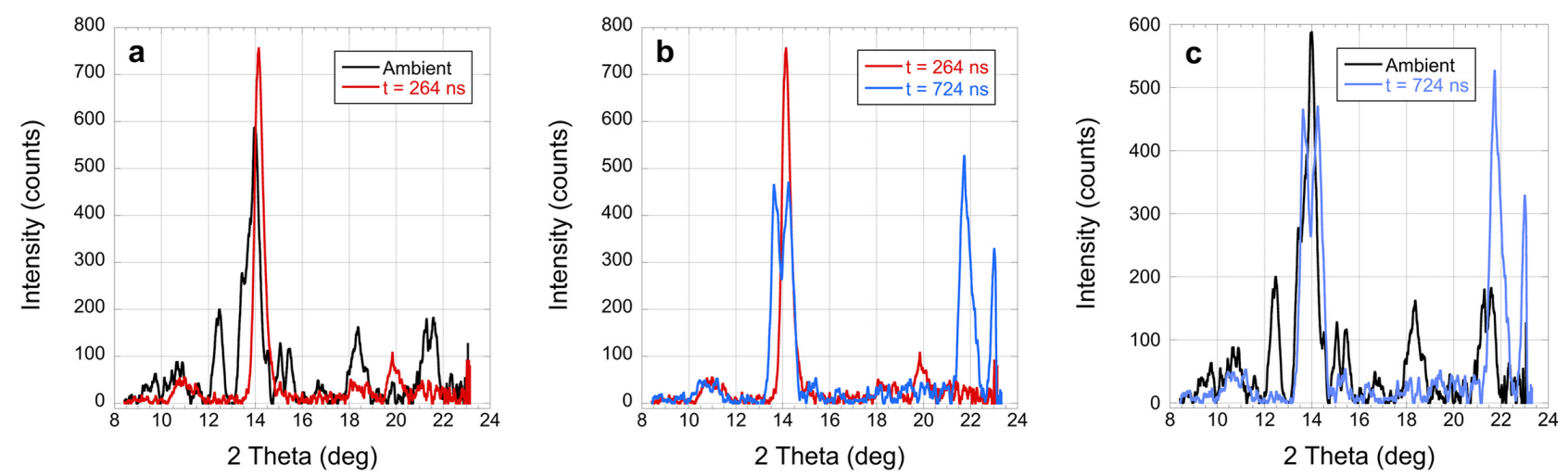

Fig. 4. XRD line profiles for test 16-1-002, showing comparisons between ambient, shocked, and partially unloaded states in titanium.

ted from analysis. Several $\alpha$-Ti peaks disappear after the initial shock, likely a result of the increased attenuation from the LiF impactor in conjunction with the short exposure time (recall that each image is formed using a single $\mathrm{x}$-ray bunch). Even so, several stronger peaks are still visible, and the overall pattern indexes as a two-phase, $\alpha-\omega$ microstructure.

It should be noted that this rapid microstructural change shown in Figure 3 is observed in the first frame after loading. For the tests presented in this work, this occurs in frame 3 of test 16-1-003, which is not pictured here, but manifests as similar to the first shocked frame in Figure 3. After the initial large-scale changes in the diffraction patterns, only subtle changes occur during the steady-state stress condition (shock state 2). Modest growth of omegaindexed peaks suggest that nucleation of omega phase happens immediately after shock-loading, followed by small amounts of growth in the omega phase. This would result in a very fragmented two-phase microstructure, lacking large, continuous, omega fragments that would be expected from a growth-dominated process. Again, the results of the present in-situ technique is consistent with previous post-mortem microstructural characterization. The two techniques are complementary, and in-situ XRD is essential to observing the kinetics of the $\alpha-\omega$ phase transition, which are impossible to infer from conventional testing and subsequent post-mortem microstructural characterization alone.

The final XRD pattern $(t=724 \mathrm{~ns})$ shows partial release (unloading) of the sample. The corresponding frame in Figure 3 (Frame 4) shows a bifurcation of the strongly contrasting ring discussed earlier, shown by arrows. Two scenarios could contribute to this behavior. The first is that some of the omega-phase material reverts back to alpha phase on unloading. The new diffraction ring would represent a significant amount of newly formed alpha phase. No significant reversion was observed in similarly shockloaded and recovered samples, however [6-8], so this scenario is unlikely to significantly contribute to the observed behavior. The second possibility is that the compressed alpha peak is overlaid with the omega peak at that d-spacing, and shifts back to the uncompressed position while the omega peak remains the same. This scenario is supported by the previous post-mortem studies. The comparison between ambient and partially unloaded states in Figure 4 shows some overlap of peaks between the initial and final states. It is challenging to fully correct for experimental factors, but analysis shows that the microstructure transforms from a single-phase, $\alpha$-Ti material to a two-phase, $\alpha-\omega$ final microstructure as a result of shock-loading.

\subsection{Selected Limitations of Technique}

As an experimental technique, in-situ XRD coupled with shock loading is an important tool to measuring kinetics and material behavior during testing, but it is not without limitations. The experimental setup is necessarily complex and the technique requires precise coordination of powder gun and x-ray systems, each individually challenging. Attenuation and absorption of x-rays by the target material can sometimes restrict testing to small specimen thicknesses (on the order of $150 \mu \mathrm{m}$, much smaller 
than conventional plate impact tests). The act of probing bulk behavior during a high-rate test requires one to sacrifice some imaging quality. This adds noise to collected XRD patterns and introduces a continuously shifting reference frame as the sample begins to move after impact. This technique is powerful, highly adaptable, and can potentially be applied to a wide array of materials science challenges, but may not be suitable for all materials and conditions. However, many of the previously stated issues can be (and have been) addressed through careful experimental planning, and advancement of light sources and techniques to achieve better imaging at short timescales. Furthermore, it is oftentimes helpful to include additional information via complementary conventional testing techniques (such as shock-recovery and microstructural characterization via electron microscopy or static diffraction) to aid in the interpretation of XRD results.

\section{Summary}

Strongly textured polycrystalline titanium was tested using x-ray diffraction paired with plate-impact shock loading with a powder gun. The resulting diffraction patterns clearly show an evolution of the microstructure as a function of time. For the first time, transformation to omega phase under plate impact conditions was observed for a single sample in-situ, during testing. A two-part material response was observed that included a massive microstructural change immediately after impact, followed by a subtle small evolution attributed to growth of omega phase regions. During unloading, diffraction peaks were observed to change positions. By comparing with previous post-mortem results, it was determined that this represents a decompression of the alpha lattice rather than a reverse transformation from omega to alpha. A small amount of heating due to the x-ray beam was noted, but this is not expected to have a large effect on material behavior, and future studies will utilize additional controls to reduce beam heating. These in-situ data shed new light on post-mortem examinations of shocked material previously performed at LANL. The mechanical response is believed to involve a complex coupling of phase transformation and traditional plasticity mechanisms, and the kinetics of these mechanisms play a large role in the material behavior under extreme conditions. Overall, the results presented here are consistent with previous results, and add important information regarding transformation behavior and kinetics not previously available.

Work was funded by Science Campaign 2 at LANL. Los Alamos National Laboratory is operated by Los Alamos National Security, LLC; for the National Nuclear Security Administration of the U.S. Department of Energy under contract DE-AC52-
06NA25396. This publication is based upon work performed at the Dynamic Compression Sector, which is operated by Washington State University under the U.S. Department of Energy (DOE)/National Nuclear Security Administration award no. DENA0002442. This research used resources of the Advanced Photon Source, a DOE Office of Science User Facility operated for the DOE Office of Science by Argonne National Laboratory under contract no. DE-AC02-06CH11357. LA-UR-18-21524.

\section{References}

1. S.G. Song, G.T. Gray, III, Philosophical Magazine, Part A 71, 275 (1995)

2. R.G. Hennig, D.R. Trinkle, J. Bouchet, S.G. Srinivasan, R.C. Albers, J.W. Wilkins, Nature Materials 4, 129 (2005)

3. P.A. Rigg, C.W. Greeff, M.D. Knudson, G.T. Gray, R.S. Hixson, Journal of Applied Physics 106, 123532 (2009)

4. E.K. Cerreta, J.P. Escobedo, P.A. Rigg, C.P. Trujillo, D.W. Brown, T.A. Sisneros, B. Clausen, M.F. Lopez, T. Lookman, C.A. Bronkhorst et al., Acta Materialia 61, 7712 (2013)

5. D.W. Brown, J.D. Almer, L. Balogh, E.K. Cerreta, B. Clausen, J.P. Escobedo-Diaz, T.A. Sisneros, P.L. Mosbrucker, E.F. Tulk, S.C. Vogel, Acta Materialia 67, 383 (2014)

6. B.M. Morrow, P.A. Rigg, D.R. Jones, F.L. Addessio, C.P. Trujillo, R.A. Saavedra, D.T. Martinez, E.K. Cerreta, Journal of Dynamic Behavior of Materials 3, 526 (2017)

7. D.R. Jones, B.M. Morrow, C.P. Trujillo, G.T. Gray, III, E.K. Cerreta, Journal of Applied Physics 122, 045902 (2017)

8. B.M. Morrow, J.P. Escobedo, R.D. Field, R.M. Dickerson, P.O. Dickerson, C.P. Trujillo, E.K. Cerreta, AIP Conference Proceedings 1793, 100033 (2017)

9. E.K. Cerreta, F.L. Addessio, C.A. Bronkhorst, D.R. Jones, T. Lookman, D.T. Martinez, B.M. Morrow, P.A. Rigg, C.P. Trujillo, Path Dependency of Phase Transformations, in AIP Conference Proceedings (in press) (2018)

10. S.N. Luo, B.J. Jensen, D.E. Hooks, K. Fezzaa, K.J. Ramos, J.D. Yeager, K. Kwiatkowski, T. Shimada, Review of Scientific Instruments 83, 073903 (2012)

11. N. Sinclair, Y. Wang, S. Turneaure, K. Zimmerman, Y. Toyoda, Y. Gupta, The Multi-Frame X-ray Diffraction and Imaging Detector at the Dynamic Compression Sector, in AIP Conference Proceedings (in press) (2018)

12. C. Prescher, V.B. Prakapenka, High Pressure Research 35, 223 (2015) 
